\title{
Bacteria causing ventriculoperitoneal shunt infections in a Kenyan population
}

\author{
Naomi Ochieng', MD, ${ }^{1,2}$ Humphrey Okechi, MBChB, ${ }^{2}$ Susan Ferson, CPNP, ${ }^{2}$ \\ and A. Leland Albright, MD²
}

${ }^{1}$ Department of Surgery, Division of Neurosurgery, University of Nairobi; and 'Department of Surgery, Division of Neurosurgery, Kijabe Hospital, Kijabe, Kenya

\begin{abstract}
OBJECT Ventriculoperitoneal shunt (VPS) infections are a major cause of morbidity and mortality in patients with hydrocephalus. Most data about these infections come from the Western literature. Few data about infecting organisms in Africa are available, yet knowledge of these organisms is important for the prevention and treatment of infectious complications. The purpose of this study was to determine the organisms cultured from infected shunts in a rural Kenyan hospital.
\end{abstract}

METHODS The authors conducted a retrospective study of patients with VPS infections recorded in the neurosurgical database of BethanyKids at Kijabe Hospital between September 2010 and July 2012.

RESULTS Among 53 VPS infections confirmed by culture, 68\% occurred in patients who were younger than 6 months. Seventy-nine percent of the infections occurred within 2 months after shunt insertion. Only $51 \%$ of infections were caused by Staphylococcus species (Staphylococcus aureus 25\%, other Staphylococcus species 26\%), whereas 40\% were caused by gram-negative bacteria. All S. aureus infections and $79 \%$ of other Staphylococcus infections were sensitive to cefazolin, but only 1 of 21 gram-negative bacteria was sensitive to it. The majority of gram-negative bacterial infections were multidrug resistant, but 17 of the 20 gram-negative bacteria were sensitive to meropenem. Gram-negative bacterial infections were associated with worse outcomes.

CONCLUSIONS The high proportion of gram-negative infections differs from data in the Western literature, in which Staphylococcus epidermidis is by far the most common organism. Once a patient is diagnosed with a VPS infection in Kenya, immediate treatment is recommended to cover both gram-positive and gram-negative bacterial infections. Data from other Sub-Saharan countries are needed to determine if those countries have the same increased frequency of gram-negative infections.

http://thejns.org/doi/abs/10.3171/2014.10.PEDS14178

KEY WORDS VP shunt infections; bacteria; antibiotics; hydrocephalus

$\mathrm{V}$ ENTRICULOPERITONEAL shunt (VPS) infections are a major cause of morbidity and mortality in children requiring CSF shunts. In the Western world, shunt infection rates have been reported to range from $3 \%$ to 9\%. ${ }^{3,4,12}$ Infection rates in African countries have been reported to be much higher: $9 \%-32 \%{ }^{5,8,10}$ Most studies from developed countries report Staphylococcus epidermidis as the most common infectious agent, followed by Staphylococcus aureus.$^{1,13}$ Similarly, in a study from Chile, Pena et al. documented the most common organism as coagulasenegative Staphylococcus, which accounted for 50\% (13 of 26) of the infections. ${ }^{11}$ Gram-negative bacteria contrib- ute to a small percentage of VPS infections in developed countries. ${ }^{13}$

Current treatment protocols in African countries are based on data from the Western literature, in which grampositive pathogens are more prevalent. However, few data on the infectious organisms causing VPS infections in Africa are available. Bokhary and Kamal from Libya, a North African country, noted a $77 \%$ incidence of gram-negative organisms as the offending agents in VPS infections. ${ }^{2}$ In light of that report, it is important to study the pattern of VPS infections in Sub-Saharan Africa. We sought to determine the spectrum of microbes cultured from patients

ABBREVIATIONS EVD = external ventricular drain; ICP = intracranial pressure; VPS = ventriculoperitoneal shunt; $W B C=$ white blood cell. SUBMITTED April 4, 2014. ACCEPTED October 13, 2014.

INCLUDE WHEN CITING Published online November 28, 2014; DOI: 10.3171/2014.10.PEDS14178.

DISCLOSURE The authors report no conflict of interest concerning the materials or methods used in this study or the findings specified in this paper. 
with VPS infections and their antibiotic sensitivities in the Sub-Saharan country of Kenya.

\section{Methods}

A pediatric neurosurgical database was used to search for all patients who had a recorded VPS infection between September 2010 and July 2012. The diagnosis of shunt infection was based on clinical presentation and CSF microbiology. Clinical features suggesting VPS infection included headaches, meningismus, lethargy, bulging fontanel, abdominal pain and tenderness, nausea, vomiting, swelling, erythema, pain and tenderness along the shunt tract, and fever. Patients with these symptoms were subjected to ventricular tap or shunt tap, depending on whether the fontanel was open or closed. Ventricular taps were preferred because they obviate the risk of the tap infecting the shunt or damaging its valve and they have minimal risk. Cerebrospinal fluid samples were taken for Gram stain, microscopic examination, and cell count. Samples of CSF with more than 4 white blood cells (WBCs) were cultured aerobically and had antibiotic sensitivity testing. Fungal and anaerobic cultures could not be done by the hospital laboratory.

In patients with a diagnosis of VPS infection based on clinical and CSF white blood cell criteria, the shunt was removed and an external ventricular drain (EVD) was inserted. The EVDs were not routinely changed unless they were blocked or accidentally pulled out. Antibiotics were given empirically until CSF culture results were available. Empirical antibiotics consisted of ceftriaxone for children older than 1 month and ampicillin and gentamycin for neonates. After CSF culture results were available (usually after 3 days), patients were switched to organism-specific antibiotics. Patients were treated for an average duration of 2 weeks, after which CSF cultures were repeated. Depending on the results of the second culture, antibiotics were discontinued, continued, or changed. Outcomes were recorded in relation to a patient's ability to clear the infection. A patient was considered to have cleared an infection once CSF cultures were negative after antibiotic treatment and CSF WBC counts decreased.

Of those who had negative CSF cultures after antibiotic treatment, some underwent placement of a second VPS during the same admission. Shunts were replaced if patients had signs of increased intracranial pressure (ICP) after their infection was cleared. If a second shunt was placed, CSF was always analyzed the day before the operation to confirm clearance of the infection. Many of the infants who had gram-negative infections did not have signs of increased ICP after their infections, presumably because the infections caused scarring of the choroid plexus, and they were sent home to return for follow-up in 2 weeks. If the same bacterial organism persisted in the CSF despite appropriate antibiotic therapy, and patients in this category were considered to have chronic ventriculitis. Those with very severe infections, that is, those with CSF WBC counts $>1000$ cells $/ \mathrm{ml}$ and no response to the antibiotics, were sent home on palliative care after counseling.

Charts of patients with recorded infections were retrieved, and data were analyzed. We did not discriminate between first shunt infections and subsequent VPS infections. Each shunt infection was considered as an individual entity. All patients with negative culture results from the first CSF specimen were excluded from the study. Those with missing data were also excluded.

Data pertaining to patient age, sex, CSF cell count, Gram stain, bacteria cultured, antibiotic sensitivities, time after VPS insertion, and outcomes were recorded. Data were entered, coded, and analyzed with SPSS statistics 17.0. Statistical analyses included frequencies and cross tabulation.

\section{Results}

Of the 127 VPS infections diagnosed using clinical and CSF WBC criteria, 53 had positive CSF cultures and thus were included in our study. The fact that 53 of 127 infections had positive cultures reflects the high rate of falsenegative cultures in our laboratory. Of these 53 infections, 46 were for initial shunts and 7 were for repeat shunts. Seven of the 53 infections had occurred in the same patient. Of the 53 cases included in the study, 25 occurred in males and 28 in females. Patient ages ranged from 1 month to 20 years, although the patient in $68 \%$ of the cases was younger than 6 months and in $81 \%$ of the cases was younger than 1 year (Fig. 1). Seventy-nine percent of the infections occurred within 2 months of shunt insertion. The majority of infections occurred within 6 months of shunt insertion. Shunt infections drastically decreased 6 months after VPS insertion, and late shunt infections were rare (Fig. 2).

As shown in Fig. 3, gram-positive organisms were isolated from $50.95 \%$ of cases and gram-negative organisms from $39.6 \%$.

All of the S.aureus and 92\% of other Staphylococcus species infections were sensitive to cefazolin. However, only 1 of the 21 infections caused by gram-negative bacilli was sensitive to cefazolin. The most effective antibiotic for gram-negative bacilli was meropenem, to which 17 (85\%) of 20 organisms were sensitive. Two gram-negative bacilli showed resistance to meropenem, and 1 had intermediate sensitivity. Ciprofloxacin was effective against 10 of 20 gram-negative bacilli for which it was tested, 7 of 20 were resistant, and 3 showed intermediate sensitivity. Gentamycin was effective in only 6 (30\%) of 20 cases of gram-negative bacilli infections. Only 2 of 8 gram-negative bacilli tested against cefepime and 2 of 8 tested against ceftazidime were sensitive to these antibiotics.

Chloramphenicol had the greatest effect on all grampositive and gram-negative bacteria, being effective in 33 (25 gram-positive and 8 gram-negative) of 51 infections. However, its effect was mainly on the gram-positive infections. Ampicillin was ineffective against gram-positive and gram-negative organisms, with sensitivity detected in only 2 of 25 and 0 of 20 organisms tested, respectively. Gram-positive organisms were not tested against ceftriaxone. Only 3 of 17 gram-negative organisms tested were sensitive to ceftriaxone (Table 1).

Infections with gram-negative bacilli were more common in neonates and were associated with worse outcomes. Only 52\% of those with gram-negative infections were cleared by antibiotics, compared with $77 \%$ and $91 \%$ of 


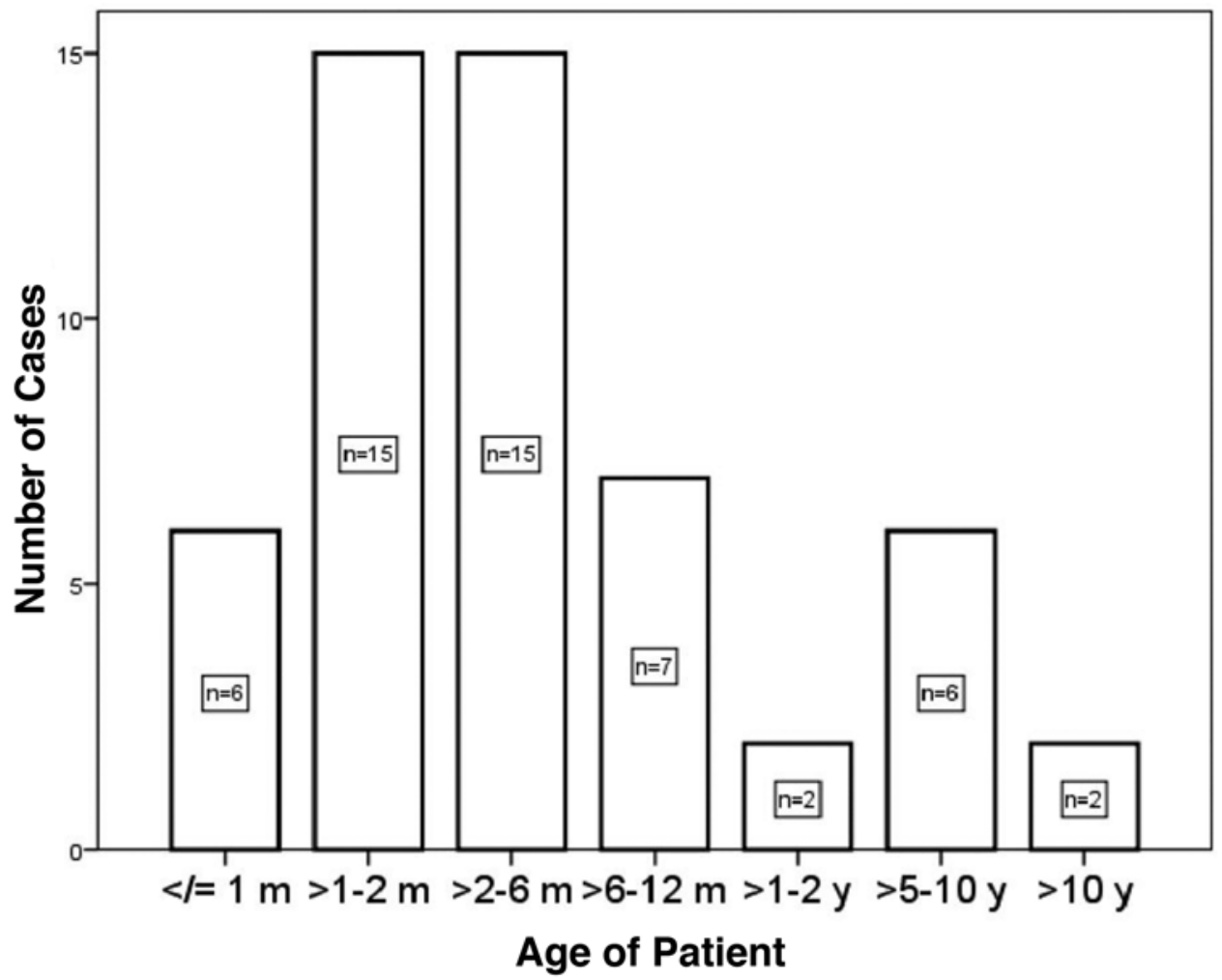

FIG. 1. Age distribution of patients with VPS infections. $m=$ month(s); $n=$ number of cases; $y=$ year(s).

those with S. aureus and other Staphylococcus species infections, respectively. Antibiotics were successful in clearing VPS infections in $68 \%$ of cases. Forty-two percent (22 of 53) of cases had a new VPS inserted after the infection cleared. There was a $4 \%$ (2 of 46 patients) mortality rate, with patients in $17 \%$ (9 of 53) of cases being sent home on

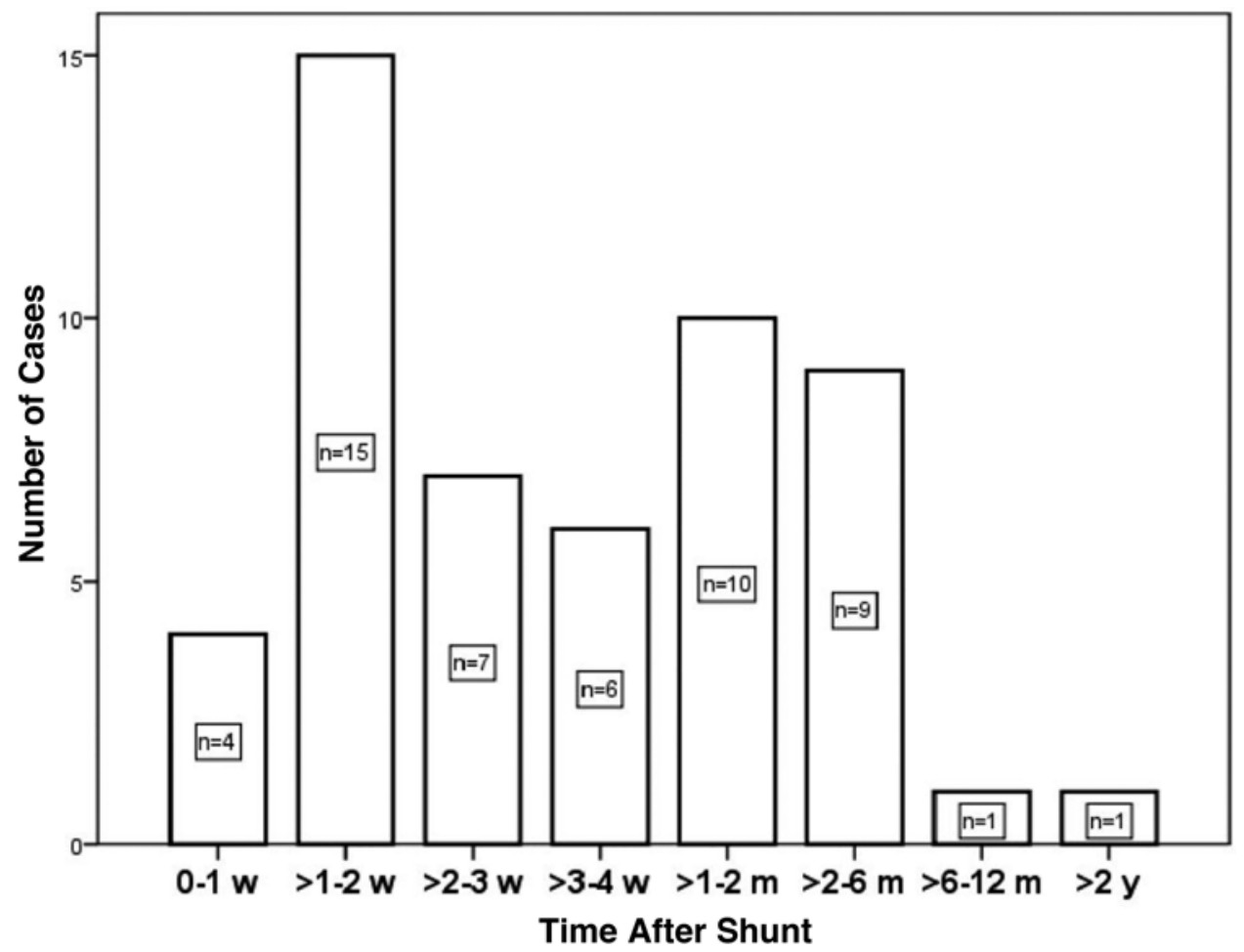

FIG. 2. Time between placement of VPS and the occurrence of shunt infection. $w=$ week(s); $m=$ month(s); $y=y e a r(s)$. 


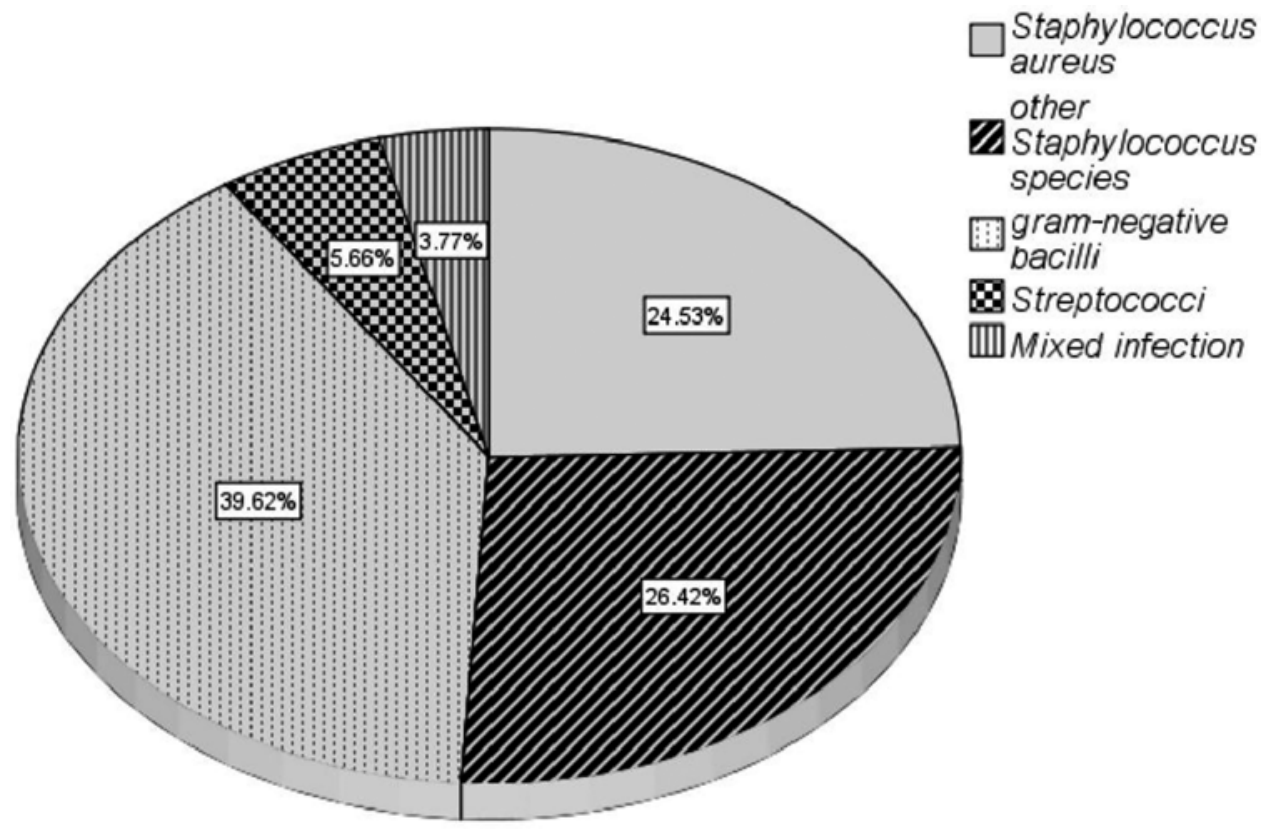

FIG. 3. Pathogens causing the VPS infections.

palliative care overall (Table 2). Five of the 9 patients sent home on palliative care had gram-negative infections.

\section{Discussion}

Ventriculoperitoneal shunt infections are a major cause of morbidity and mortality in children requiring CSF diversion. Our study comprised a young population, ranging in age from less than 1 month to 20 years with a mode of 2 months and a median of 4 months, and establishes gramnegative bacilli as a major causative pathogen.

\section{Time of Infection}

Most infections occurred within the first 2 months of shunt insertion; $60 \%$ of these occurred within the first month. This is in keeping with what is reported in the literature. ${ }^{69}$ Lima et al. reported the average time to shunt infection diagnosis as 26 days after VPS insertion, and $81 \%$ of infections in their study occurred within 1 month. ${ }^{9}$

In our study, a peak incidence of VPS infections occurred in the 2 nd week postinsertion, which may represent early colonization of the shunt during the perioperative period. Patients presenting with illness more than 6 months

TABLE 1. Antibiotic sensitivity of pathogens

\begin{tabular}{|c|c|c|c|c|c|}
\hline \multirow[b]{2}{*}{ Antibiotic } & \multicolumn{5}{|c|}{ No. of Pathogens Sensitive to Antibiotic/No. Tested } \\
\hline & Staphylococcus aureus & Other Staphylococcus spp & Gram-Negative Bacilli & Mixed Infections & Total \\
\hline Ciprofloxacin & $0 / 1$ & $0 / 0$ & $10 / 20$ & $3 / 4$ & $13 / 25$ \\
\hline Meropenem & $2 / 2$ & $5 / 5$ & $17 / 20$ & $4 / 4$ & $28 / 31$ \\
\hline Tetracycline & $8 / 11$ & $6 / 12$ & $5 / 18$ & $0 / 5$ & $19 / 46$ \\
\hline Gentamycin & $0 / 0$ & $0 / 0$ & $6 / 20$ & $1 / 3$ & $7 / 23$ \\
\hline Tazobactum & $1 / 2$ & $2 / 4$ & $4 / 12$ & $1 / 3$ & $8 / 21$ \\
\hline Chloramphenicol & $13 / 13$ & $12 / 14$ & $6 / 19$ & $2 / 5$ & $33 / 51$ \\
\hline Erythromycin & $8 / 13$ & $7 / 12$ & $0 / 0$ & $1 / 3$ & $16 / 28$ \\
\hline Cefazolin & $12 / 12$ & $11 / 12$ & $2 / 18$ & $2 / 4$ & $27 / 46$ \\
\hline Ampicillin/sulbactum & $0 / 0$ & $0 / 0$ & $5 / 5$ & $0 / 0$ & $5 / 5$ \\
\hline Ceftriaxone & $0 / 1$ & $0 / 0$ & $3 / 17$ & $2 / 4$ & $5 / 22$ \\
\hline Cotrimoxazole & $7 / 11$ & $0 / 10$ & $1 / 15$ & $2 / 6$ & $10 / 42$ \\
\hline Lincomycin & $9 / 13$ & $6 / 12$ & $0 / 0$ & $0 / 2$ & $15 / 27$ \\
\hline Oxacilin & $5 / 13$ & $2 / 12$ & $0 / 0$ & $0 / 2$ & $7 / 27$ \\
\hline Ampicillin & $1 / 13$ & $1 / 12$ & $0 / 20$ & $1 / 4$ & $3 / 49$ \\
\hline Cefepime & $0 / 0$ & $0 / 0$ & $2 / 8$ & $0 / 0$ & $2 / 8$ \\
\hline Total no. of pathogens tested & 13 & 14 & 20 & 6 & 51 \\
\hline
\end{tabular}


TABLE 2. Outcomes of VPS infections based on pathogens grown

\begin{tabular}{lccccccc}
\hline & \multicolumn{1}{c}{ No. of Patients } \\
\cline { 2 - 8 } \multicolumn{1}{c}{ Pathogen } & $\begin{array}{c}\text { Cleared } \\
\text { Infection }\end{array}$ & $\begin{array}{c}\text { Cleared Infection } \\
\text { \& VPS Replaced }\end{array}$ & Death & $\begin{array}{c}\text { Palliative } \\
\text { Care }\end{array}$ & $\begin{array}{c}\text { Chronic } \\
\text { Ventriculitis }\end{array}$ & $\begin{array}{c}\text { Growth of Other } \\
\text { Pathogen }\end{array}$ & $\begin{array}{c}\text { Total } \\
\text { Staphylococcus aureus }\end{array}$ \\
\hline Other Staphylococcus spp & 5 & 5 & 0 & 2 & 1 & 0 & 13 \\
\hline Gram-negative bacilli & 2 & 9 & 0 & 1 & 0 & 0 & 12 \\
\hline Streptococcus spp & 5 & 6 & 1 & 5 & 1 & 3 & 21 \\
\hline Mixed infection & 1 & 1 & 1 & 0 & 0 & 0 & 3 \\
\hline Total & 0 & 1 & 0 & 1 & 0 & 0 & 2 \\
\hline
\end{tabular}

after shunt insertion should be examined for other causes, such as urinary tract infections, before one concludes that there is a CSF infection.

\section{Spectrum of Infecting Pathogens}

We found a high incidence of gram-positive bacterial infections; Staphylococcus species combined accounted for slightly more than $50 \%$ of the infections. Though this rate is lower than the $75 \%$ incidence reported in the Western literature, ${ }^{12}$ the number is significant.

Forty percent of infections were attributable to gramnegative bacilli, contrary to the $6.1 \%$ rate reported in the literature by Stamos et al. ${ }^{13}$ However, our observation that there is an increased frequency of gram-negative infections is consistent with the findings of Bokhary and Kamal in Libya, where gram-negative bacilli accounted for $77 \%$ of the infections. ${ }^{2}$ In that study, most gram-negative bacilli infections occurred in patients with hydrocephalous due to intraventricular hemorrhage or meningitis. Most of the patients in our study had hydrocephalous caused by spina bifida or meningitis. Bokhary and Kamal postulated that the high incidence of gram-negative bacilli in their population was caused by the empirical use of second- and third-generation cephalosporins. The high incidence of gram-negative bacilli in our population could be attributable to malnutrition, poor hygiene postoperatively, and previous exposure to antibiotics, especially in patients who had hydrocephalus in association with spina bifida. Our hospital's environment may also contribute to the high frequency of gram-negative infections. The frequency of such infections is high in other areas of the hospital (for example, the men's, women's, and pediatrics wards), which is partly attributable to patients being crowded together 8-9 people in a room, the difficulty in isolating patients with documented gram-negative infections, and the different levels of hygiene in the hospital (that is, linens and gowns changed infrequently and linens washed in cold water rather than hot).

The shunt techniques used in Kijabe were similar to those used in the US, although a defined protocol was not used. Most patients had a chlorhexidine bath on the morning of their operation. After the induction of anesthesia, scalp hair was trimmed with clippers and was never shaved with a razor. Skin was cleaned with alcohol-soaked gauze and then washed with chlorhexidine. Betadine was used as the final prep solution. Intravenous cefuroxime $(50 \mathrm{mg} / \mathrm{kg})$ was given before skin incision. Double gloving was always used. The surgical field was covered with Ioban drapes. Cefuroxime was given every 8 hours, for 3 doses postoperatively. Chhabra shunts (Surgiwear Inc.) were used in most patients. No antibiotic-impregnated shunts were used. Thus, shunt techniques are unlikely to be related to the high frequency of gram-negative infections.

\section{Antibiotic Sensitivities}

Most gram-negative bacilli were multidrug resistant, responding to only 1 or 2 drugs. Meropenem was the most effective antibiotic against gram-negative bacilli. It is concerning that 2 gram-negative bacilli were resistant to meropenem and that 1 had only intermediate sensitivity. In view of the risk of introducing meropenem resistance and given its relative high cost, it would be prudent to seek another antibiotic for use and to reserve meropenem as a last resort. Ciprofloxacin had the second-best coverage of gram-negative bacilli in our series and may offer an alternative to meropenem for empirical treatment. There is, however, a relative risk of epiphyseal damage with ciprofloxacin administration during early infancy, but previous studies set a precedent for using it and also demonstrated its effectiveness in treating neonatal gram-negative meningitis. ${ }^{7}$ Given the difficulty in clearing gram-negative bacilli infections (clearance of $52 \%$ in our study) and the emerging risk of meropenem-resistant gram-negative bacilli, it may be advisable to use antibiotics of 2 different classes to which the organism is sensitive that have good CSF penetration.

Aminoglycosides and second- and third-generation cephalosporins, which are widely used, were ineffective against gram-negative bacilli, an observation that differs from the findings of Pena et al. in which Klebsiella species and Escherichia coli were sensitive to third-generation cephalosporins and aminoglycosides. ${ }^{11}$ All Staphylococcus species showed sensitivity to cefazolin, which has poor CSF penetration and hence cannot be used to treat VPS infections. However, it can be used for preoperative prophylaxis to act against skin commensals. Pena et al. found that the use of preoperative cefazolin was not protective against VPS infections, although their patients had a high incidence of methicillin-resistant $S$. aureus, which was not the case in our study. Chloramphenicol, which is rarely used in Kenya, was found to be effective against gram-positive infections and some gram-negative infec- 
tions. It has good CSF penetration and may provide good gram-positive coverage for the empirical treatment of shunt infections.

Most organisms were resistant to ampicillin and gentamycin. This fact, combined with the higher prevalence of gram-negative infections in neonates, would explain the poorer outcomes in the patients in this age group, who received ampicillin and gentamycin as empirical therapy for shunt infections.

\section{Conclusions}

Gram-negative bacilli shunt infections are much more common in Kenya than has been reported in the literature from Western countries. Gram-negative bacilli infections had worse outcomes than gram-positive bacterial infections. This result could be attributable to the delayed treatment with appropriate antibiotics and the occurrence of these infections in neonates who have lower immunity. Most of the patients with gram-positive bacterial infections had good outcomes, as most had antibiotics that were effective from the very start.

In Sub-Saharan Africa, it is imperative to start any patient suspected of having a VPS infection on antibiotics that cover both gram-positive and gram-negative bacteria while CSF culture results are pending. We recommend antibiotic treatment with chloramphenicol and ciprofloxacin until CSF cultures are available and antibiotic sensitivity is known.

\section{Acknowledgments}

We gratefully acknowledge Mr. Wycliffe Ayieko, who aided in the data analysis, and the International Brain Research Organization (IBRO) workshop on writing skills held in Rabat, Morocco.

\section{References}

1. Al-Dabbagh M, Dobson S: Management of shunt related infections. Adv Exp Med Biol 719:105-115, 2011

2. Bokhary MA, Kamal H: Ventriculo-peritoneal shunt infections in infants and children. Libyan J Med 3:20-22, 2008

3. Borgbjerg BM, Gjerris F, Albeck MJ, Børgesen SE: Risk of infection after cerebrospinal fluid shunt: an analysis of 884 first-time shunts. Acta Neurochir (Wien) 136:1-7, 1995

4. Cochrane DD, Kestle JR: The influence of surgical operative experience on the duration of first ventriculoperitoneal shunt function and infection. Pediatr Neurosurg 38:295-301, 2003
5. Gathura E, Poenaru D, Bransford R, Albright AL: Outcomes of ventriculoperitoneal shunt insertion in Sub-Saharan Africa. J Neurosurg Pediatr 6:329-335, 2010

6. George R, Leibrock L, Epstein M: Long-term analysis of cerebrospinal fluid shunt infections. A 25 -year experience. J Neurosurg 51:804-811, 1979

7. Kaguelidou F, Turner MA, Choonara I, Jacqz-Aigrain E: Ciprofloxacin use in neonates: a systematic review of the literature. Pediatr Infect Dis J 30:e29-e37, 2011

8. Lamprecht D, Schoeman J, Donald P, Hartzenberg H: Ventriculoperitoneal shunting in childhood tuberculous meningitis. Br J Neurosurg 15:119-125, 2001

9. Lima MM, Pereira CU, Silva AM: [Ventriculoperitoneal shunt infections in children and adolescents with hydrocephalus.] Arq Neuropsiquiatr 65:118-123, 2007 (Portuguese)

10. Mwachaka PM, Obonyo NG, Mutiso BK, Ranketi S, Mwang'ombe N: Ventriculoperitoneal shunt complications: a three-year retrospective study in a Kenyan national teaching and referral hospital. Pediatr Neurosurg 46:1-5, 2010

11. Pena AA, Sandia ZR, Riveros PR, Salazar ZC, Herrera OR, Verqara FR: [Risk factors for ventricular peritoneal shunt infection in pediatric patients from the Hospital Carlos Van Buren.] Chilean J Inf 29:38-43, 2012 (Span)

12. Simon TD, Hall M, Riva-Cambrin J, Albert JE, Jeffries HE, Lafleur B, et al: Infection rates following initial cerebrospinal fluid shunt placement across pediatric hospitals in the United States. Clinical article. J Neurosurg Pediatr 4:156-165, 2009

13. Stamos JK, Kaufman BA, Yogev R: Ventriculoperitoneal shunt infections with gram-negative bacteria. Neurosurgery 33:858-862, 1993

\section{Author Contributions}

Conception and design: Albright, Ochieng'. Acquisition of data: Ochieng'. Analysis and interpretation of data: all authors. Drafting the article: Ochieng'. Critically revising the article: Albright, Ferson. Reviewed submitted version of manuscript: all authors. Approved the final version of the manuscript on behalf of all authors: Albright. Statistical analysis: Ochieng'.

\section{Supplemental Information \\ Previous Presentation}

Portions of this work were presented in poster form at the World Society for Stereotactic and Functional Neurosurgery Quadrennial Meeting held in Tokyo, Japan, on May 27-30, 2013.

\section{Correspondence}

A. Leland Albright, Kijabe Hospital, P.O. Box 20, Kijabe 00220, Kenya.email: 1.albright@neurosurgery.wisc.edu. 